\title{
INFLUENZA DEL VENTO SLL, FUNZIONAMENTO DELLE SONDE RADIOATTIVE
}

\author{
R. Gialdea - A. Lo Surdo - G. Zanotelli
}

Fin dai primi tentativi di impiego delle sostanze radioattive per la misura del potenziale elettrico atmosferico, era stato notato (') che $\mathrm{i}$ movimenti dell'aria vi potevano avere una qualche influenza: esperienze successive $\left({ }^{2}\right)$ non hanno sempre confermato tale effetto. Una attenta osservazione di alcuni recenti rilevamenti del potenziale elettrico atmosferico, eseguiti con sonde radioative negli Oservatorii dell'Istituto Nazionale di Geofisica, ei convinsero però della notevole importanza di tale influenza, indubbiamente esistente.

$\mathrm{Ci}$ è sembrato perciò interessante eseguire ricerche sperimentali e teoriche tendenti a chiarire la questione. Abbiamo condotto a questo scopo in laboratorio misure di potenziale con sonde radioattive nell'interno di un condensatore piano, nel quale fluisce, in direzione del campo elettrico, una corrente di aria a velocita regolabile ed uniforme. E' stato così possibile rilevare l'esistenza di una relazione caratteristica tra la velocità del vento $e$ il potenziale misurato, e di un intervallo di velocità in cui lo scarto del potenziale misurato da quello statico può diventare notevole.

Per interpretare $\mathrm{i}$ risultati di queste esperienze abbiamo poi elaborato una trattazione teorica, che rappresenta nelle linee generali il fenomeno e ne giustifica qualitativamente l'andamento.

La disposizione sperimentale da noi usata per queste ricerche $\dot{e}$ costituita (fig. 1) da due grandi armature piane $A$ e $B$ di rete metallica disposte parallelamente ad una distanza di $60 \mathrm{~cm}$, fra le quali si applica una differenza di potenziale, onde avere nello spazio intermedio un campo elettrico che si può considerare uniforme nella parte centrale; in tale regione fluisce, lungo le linee del campo e su un: estensione relativamente grande $(40 \mathrm{~cm}$ di diametro), una corrente

(1) F. Henning, 'Ann. der Physik, 7, p. 893 (1902).

(2) M. Moulin, Le Radium, 4, p. 6 (1907); M. A. Tuve-C. Huff., Terr. Magn.. 32 , p. 17 (1927). 


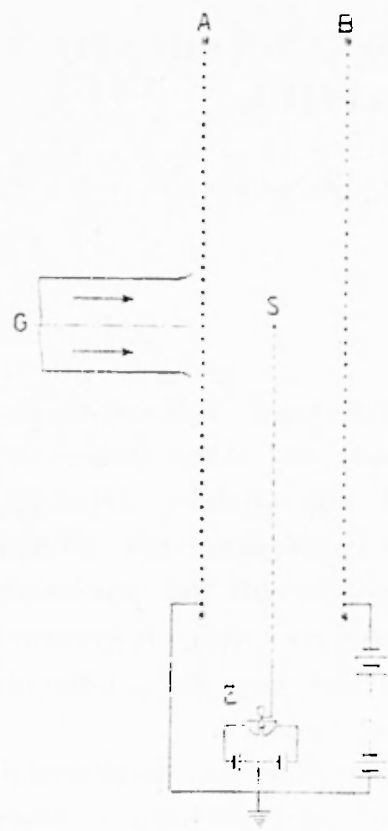

liig. 1

d"alia, li velocila praticamente miforme: la -lessa provience, allraveroo la rele melallica. dallo shoereo di mal Ealleria acerodinamien (;. La velocila dell"aria può a. -ere rarialia ron continuita e rontrollata mediante un appo-ilo anemometro a filo rallo.

la smata radiontlisa implegalta iv der lusuale lipo a di-ro (diamelro 12 mm) ron una delle facee allisala mediante polonio e semea alem ridulore di pereor-o (3. I.a vorela $S$ i collocala nella parte ecentrale del campo elettrico e debla roprente d'aria. con la slyperferie alliva nolmale al piano delle armalure.

le reperienze sono state eondotle con velociti s del vento repesente dal valore is rirea $i 30 \mathrm{~cm} / \mathrm{sec}$; la differenza di potenziale appliabal tra le armalure slesse a di 3010 roll: Larmatura a polenziale pii

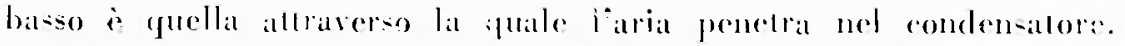

Le misure sono state fatle rolloeansto lat somda surec-ivamente in

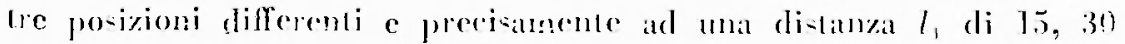

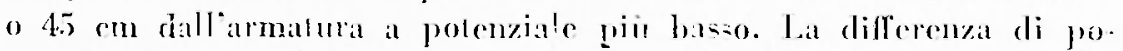

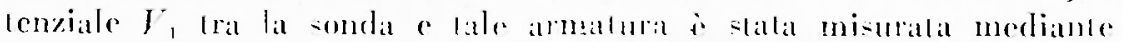
in allromeino $E$.

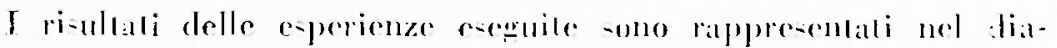

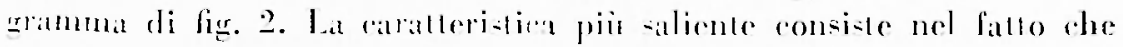
lat rarizione apportalia dallazione del renlo al polenziale, e quindi

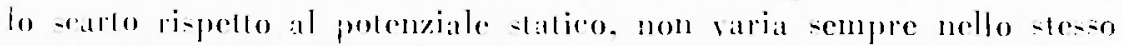
-en-a al variare della velorila del renlo. ma a partio da velocita

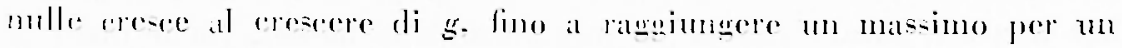

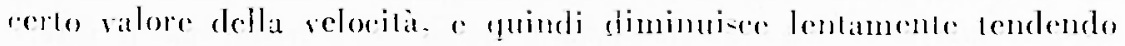
a-intoliramente a zero.

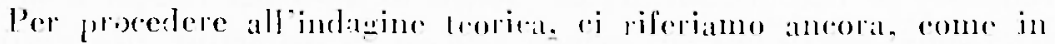

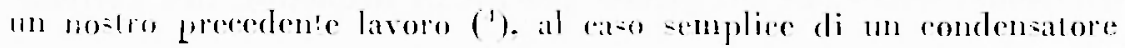

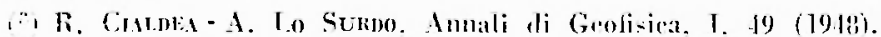

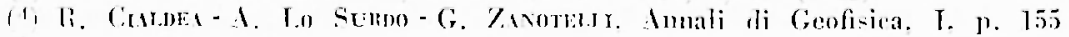
110101. 
di sezione indefinila. le rei ammature piane e parallete -ono manle. nute a dilferenza di polenziale costanle: fra fueste i compureo unn stralo piano alle slese parallelo. pure indefnilo. esoltopo-to ad aximme ionizzanle uniforme. del quale -upporremo trasurahile to spessole. Faremo astrazione dal fonomeno dellat difisione.

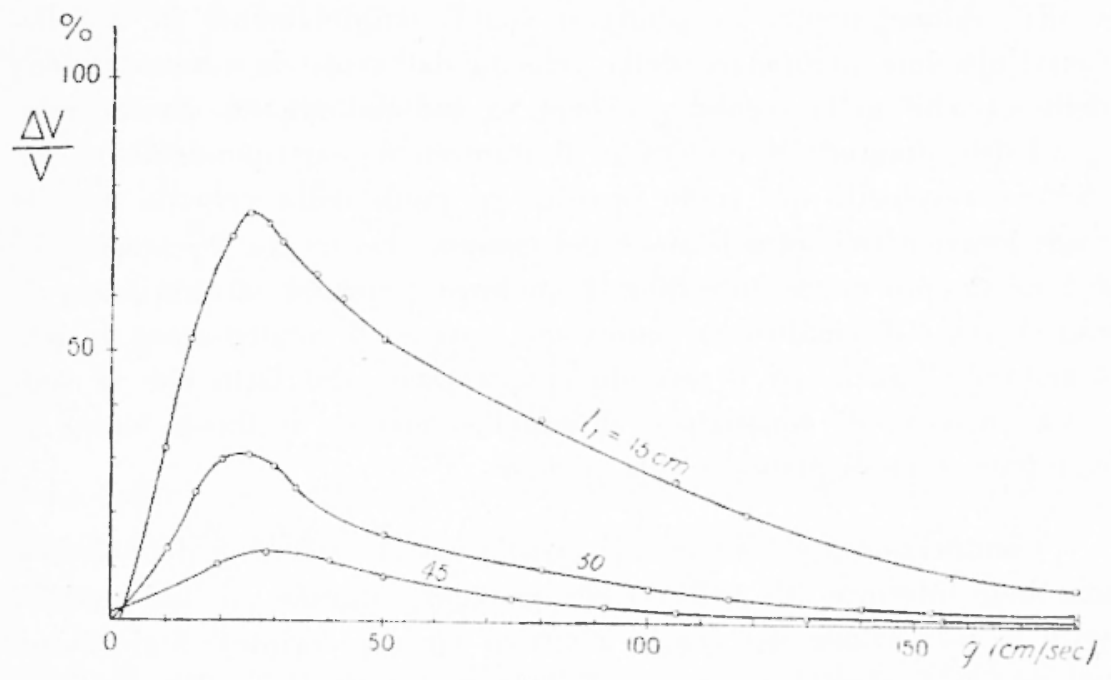

Fig. 2

Immaginiamo ahe normalmente alle ammalme allo - llalo solto-

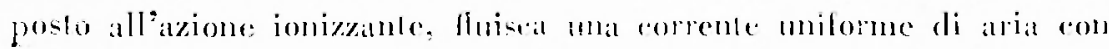

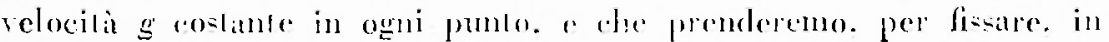

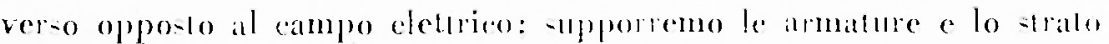

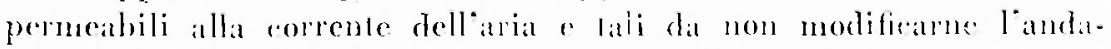
menlo.

Il problema dhe ei proponianme romsi-le nol deleminatre il pulemziale di equilibrio as-smlo dallo tralo inlermedio. Le carallesi-

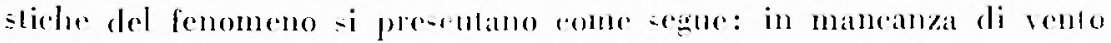
gli ioni prodolli nello strato inlermedio i dirigrono, sollo lazione del campo. i posilivi rerso ma delle monalure ed i negalivi rerso laltra. in modo rhe lo spazio inlerno del conden-alore viene divi=a dislle slabe in due regioni, la prima rontenente solo cariehe posilive e la seconda solo rarliche negalive. Per velocila relativamente piecole. il

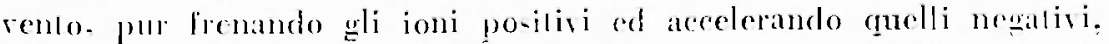
non muta -ostanzialmente que-la di-lriburione. - non nel fallo rhe gli 


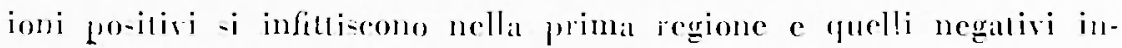
reee -i diradimo nella seconda. Pue-ta distribuzione di cariche si manliene fino a quando la relocila del rento si avvieina alla relocita ele -ollo lazione del rampo pos-ono assumere gli ioni positivi.

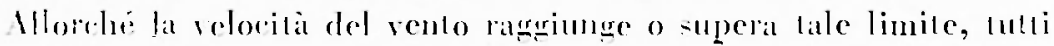

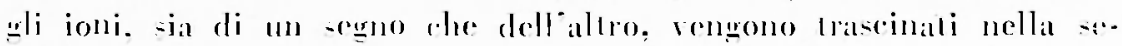

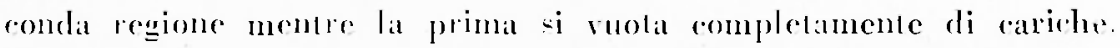
Con luberiore aumentare della velocila del vento la concentragione delle rarielie nella -eronda regione va indefinitamente diminuendo.

Esi-te dungue un intervallo di transizione corrispondente ai va. lori della relorita del rento prossimi a quelli della relocisa assumb dagli ioni posilivi sollo lazione del rampo, cle separa due regini di

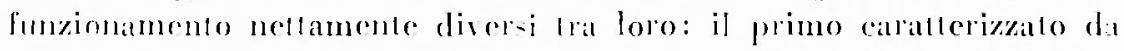

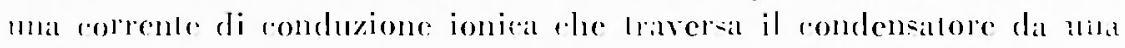
armalura allatas, od il secondo camerterizalo dal fatlo che la sudfella corente di ronduzione si amulla, mentre il flusso ionieo -i mataiene soltanto nella seconda regione.

Cominciano a considerare il raso in cui la relocita gr del vento i mantiene inferiore alle velocila rhe possono asumere gli ioni posilivi solto la obla axione del rampo eleltieo (primo regime). Stabilibemo nel nostro conden-atore un asse delle asci-se $x$ normale alle armature el arme lorigine sullo - frato ionizamle (fig. 3); la prima armalura,

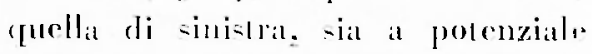

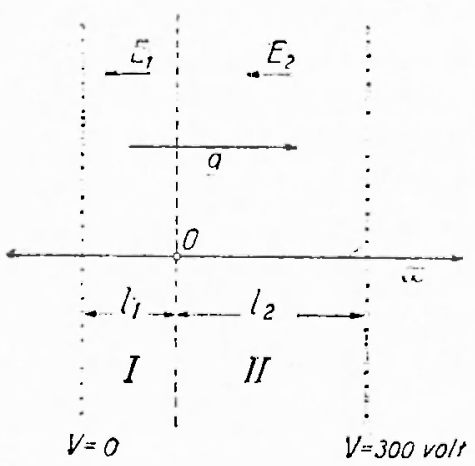

I i w. : zero: c la seronda, fuella di de-trat. a polencials $r$ positivo e costante. Il rampo eleltrico $E$ risulta quindi dirells in rerso opposto a quello positivo delle $x$ : laria invere lutiore. seconder quanto soplar dello, da sisi=lra a destra, rioe in verso conrateio. Per la romdizione imposta al valore di $g$ nella regione a sini-lrat dello -lrallo coltopo-lo all'axione ionizzante. ore $x<0$, si lrovano sollane lo joni positivi i quali -i muoromo nel verso del rampo: nella secondat renione inveres a destra dello tralo intermedio, per la quate $x>0$. a trovano -0 lo ioni negalivi i guali $\mathrm{ki}$ muovono nel verse

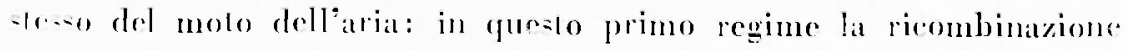


al di fuori dello slrato secle dellazione ionizzante non ha luowo. montre i verifiea nell"interno di detto strato.

Vella prima regrione $(x<0)$ e nella seconda $(x>0)$ le velociti degli ioni posilivi e rispettivamente negalivi sono esplesse. in valore e segno. da $k E+g$, ove con $k$ si $a$ indieala la mobilita degli ioni con il proprio segno. Quindi tra il cimpo elettico $E$, la concentrizione $n$

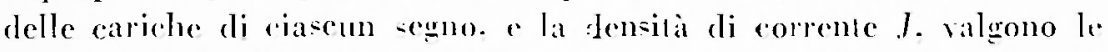
relazioni

$$
\frac{d E}{d x}=4 \pi e n \quad, \quad J=e n(k E+g)
$$

in eni e rapplesenta, in valore e segno, la carica ionica. Eliminando la $n$ tra le clue precedenti relazioni. si olliene

$$
\frac{d E}{d x}=\frac{4 \pi J}{l E+g} .
$$

In condizioni stazionarie la $J$ is costante in ogni sexione del roudensalore c ruindi indipendente dalla $x$ integrando si ha

$$
I^{2} E^{2}+2 g E-8 \pi J x-B=0
$$

esscuclo $B$ la costante di interrazione, che si può determinare ficendo $x=0$; indieando con $E_{n}$ il valore assolulo del campo in tale punto, i ha allora escendo il campo divello in verso opposto allat $r$,

$$
B=h_{i} E^{\circ}-2 g E_{0} ;
$$

sostituendo nella $[3]$ e risolvendola. si ha

$$
E(x)=-\frac{g}{k}-\sqrt{\left(F_{n}-\frac{g}{h}\right)^{2}+\frac{8 \pi J x}{k}} .
$$

Dei due segni del radicale dere essere preso il segno negativo, ar-

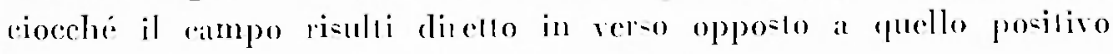
rlelle $x$ -

Esanduiamo l'andanento del rampo eleterien nelle dne regioni:

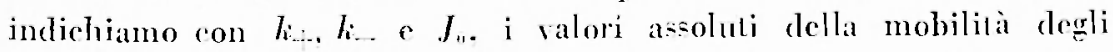
ioni a della densila di corrente. Vella prima regione. per $x<0$. axremo

$$
E_{1}(x)=-\frac{g}{k_{+}}-\sqrt{\left(E_{1,0}-\frac{g}{k_{+}}\right)^{2}-\frac{8 \pi J_{0}: x}{k}}
$$


Il rampo crese in valore aseoluto all'aumentare della distanza dall, stralo intermedio, e ciò in maniera tanto più sensibile per quatto maggiors è la g.

Nella seconda regione, per $x>0$, artemo

$$
l_{w}(x)=\frac{g}{l_{i}}-\sqrt{\left(E_{2,0}+\frac{\tilde{g}}{l_{i}}\right)^{2}+\frac{\mathbf{8}_{-T} J_{0} x}{l_{i}}}
$$

Il campo creace in valore assoluto all'aumentare della distanza dalla strate intermedio, e ciò in maniera lanto piu sensibile, cquanto mi. nole è la g.

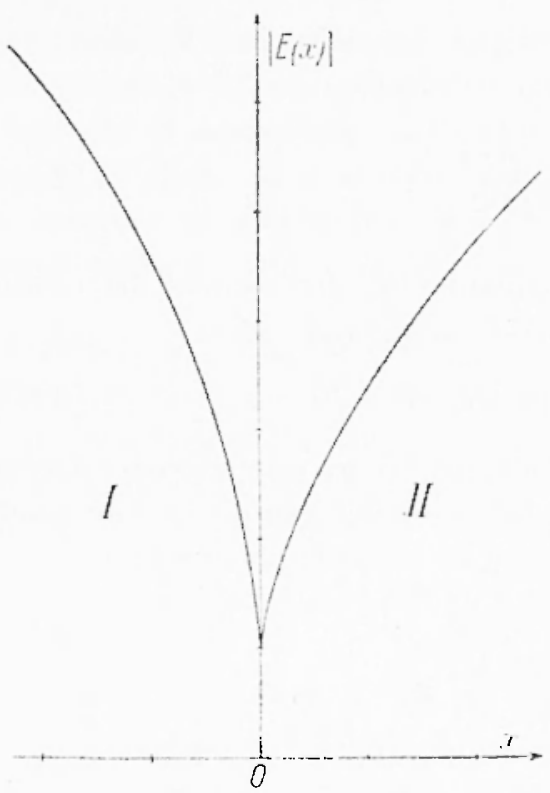

Fia. 4

Sel diatgramma di fiğ. 4 i rappresentato rualitalivament. l'andianento del rampo E' per un erto valore dellat $g$, cisendosi supposto $E_{\text {u }}$ uguale sultr. due faceie dello strato interme. dio. cioi

$$
E_{1,0}=E_{n, n}=E_{n}: \quad 8
$$

it ramo della curval a -ini-tria (prima regione) diventa pii ripiclo e quello a destra invece (cconda regione) meno. all'aumentare di g.

Quanto alla rondizione l: ahe nel seguito della tratlazione noi asumeremo rome ralida, osecriamo elo la iessa cquivale at ammettere. in base al teorema di Gauss, dic sullo stralo sede dell'azione ioni\%.

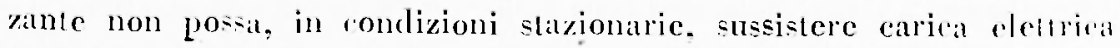
prevalentemente di un solo segno.

Oerore ora determinare la differenzat di potenziale $F$, tra lo strato e l'armatura di sinistra del endensitore; incerando la $[6]$ trá i limiti $x=0$ e $x=-l_{1}$ si ha

$$
F_{1}=\frac{1}{l_{i}}\left\{g l_{1}+\frac{1}{12 \pi J_{0} l_{i}}\left(\left[\left(E_{0} l_{i}-g\right)^{2}+8 \pi J_{0} l_{1} k\right]^{\frac{3}{2}}-\left[E_{0} k-g\right]^{3}\right)\right\}
$$




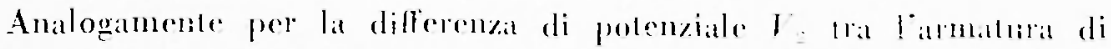

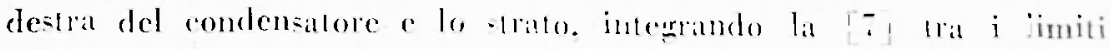
$x=l$, a $x=1)$, si hat

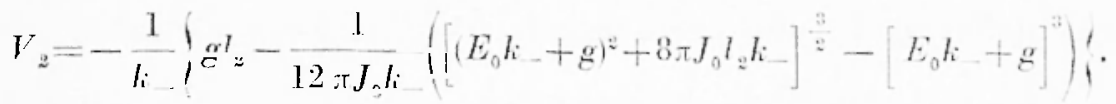

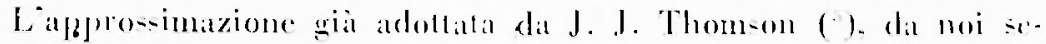
genita nel peecedente lasoro. verra qui rilenuta valida per piecole ve-

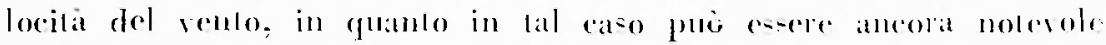
l'eflete della ricombinazione nelleinterno dello -tralo -ede della ionizzazione: in que-ta apposimatone -i ammelte come lat-curabile il

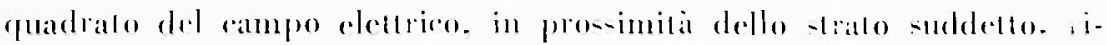

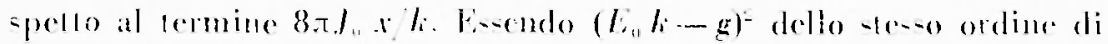

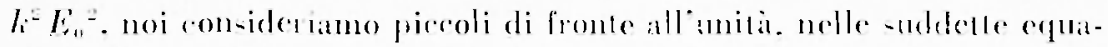

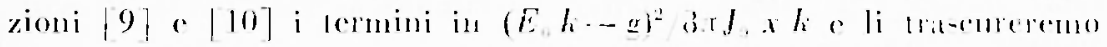

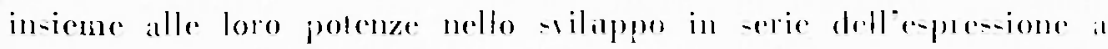

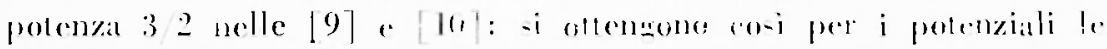
relasioni -empliliate

$$
\begin{aligned}
& V_{1}=\frac{1}{k_{1}}\left[\frac{2}{3} \sqrt{8 \pi J_{0} l_{1}^{3} k_{1}}+g l_{1}\right], \\
& V_{2}=\frac{1}{k_{i}}\left[\frac{2}{3} \sqrt{8 \pi J_{0} l_{0} k_{1}}-g l_{i}\right] .
\end{aligned}
$$

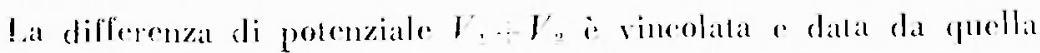
$f$ costante applicata fra le ammature del condensalore: imponembo questa uleriore condizione en eliminande la I si hat per la differenzal

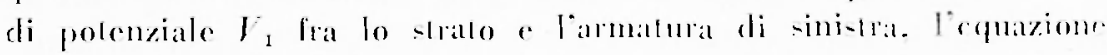

$$
V_{1}=\frac{l}{1+\sqrt{\frac{l_{2}}{l_{i}}\left(\frac{l_{2}}{l_{1}}\right)^{: 3}}}\left[1+\frac{g !_{2}}{l_{i} l}\left(1+\sqrt{\frac{l_{-} l_{2}}{l_{i} l_{1}}}\right)\right] .
$$

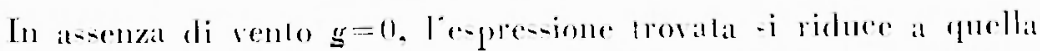
da noi gria data nel percedente lavoro, allorehe con-iderammo leftedlo della sola carica spaziale ("). La differenza di potenziale fra lo -trato e la seconda almaluma ri-ula

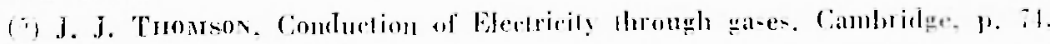
1903.

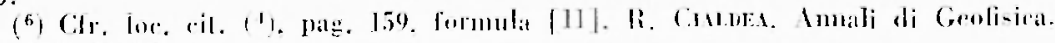
I. p. $36.1(1.196)$. 


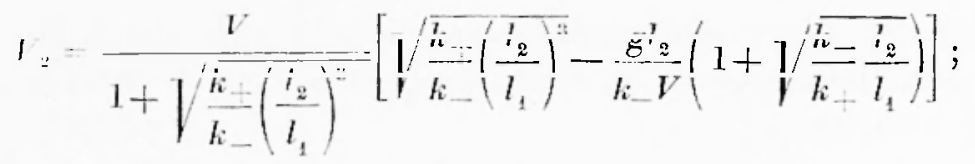

Lale differenzal di potenziale si anmulla allorehé $g$ assume il valore

$$
g_{1}=\frac{l_{i} V}{l,} \frac{1}{1+\sqrt{\frac{h_{+}+\frac{l}{1}}{l_{-} l_{2}}}}
$$

In eorri-pundenza is ha $\Gamma_{1}=\boldsymbol{I}$.

Per questa velocila git lo slralo soggello allozione ionizzanle as- mone il polenziale della seconda smalus'a, a la differenza di polenziale applicata $V$-i trova lonalizzals per intero fra dello slralo e la prima armalma. Valori magriori non sono ammissibili, perehé porlerebbero ad una differenza di potenziale piò mrande di quella appli"ala: il valore s, limila dumfue superiomente, dal pumbo di vi-la analilico. l"inlervallo di variazions della g nel primo leegime.

Comsideriamo ora il aso in eni ha velorila g del rento sia sempre -uperiore alle volociti cle posono aggrimgere gli ioni positivi sollo lit ola azione del campo chellrieo (secondo regime). Per la condizione re-i imposta al ralore di lale velocila, la prima regione rimane vuola

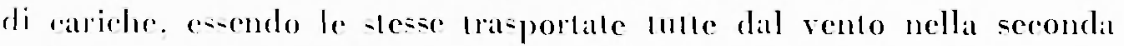
renione: in fues "ultima gli ioni posilivi e negativi si muovono ambedue nello seso veroo, i negatlivi aneclerati, i positivi mallentati dal

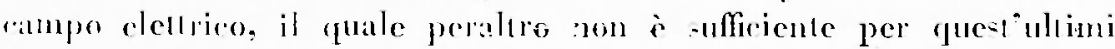
at vincere l'azione di traseinamento dell'aria.

Vellia econda regione la velocila degli ioni positivi e espressa da $g+k$ E r quella dei negalivi da $g-h-E$. ambedue maggiori di zero.

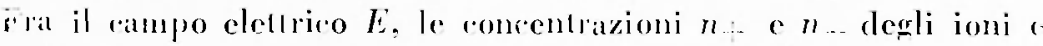
lit dentia di comenle di riasenn segno $/$ r $J$.. valgono le relazioni

$$
\begin{gathered}
\frac{d E}{d x}=4 \pi(e n+e n) . \\
J=e n(g+k E)>0, \quad J=e n(g-h \quad E)<0 .
\end{gathered}
$$

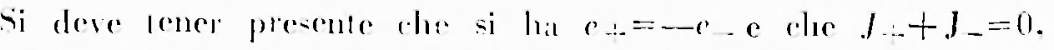
peiche in eondizioni sazionaric, mancando le cariche nella prima regione. dere anede nulla anche la coriente altraverso la seconda resiones. Litiminando le conecontrazioni fra le relaxioni precedenti. pre.

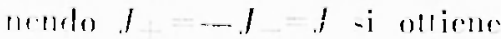




$$
\frac{d E}{d x}=4 \pi J\left[\frac{1}{g+k \ldots E}-\frac{1}{g-k_{i} E}\right] .
$$

Anche in yuesto caso la $l$, in condizioni stazionarie. is indipendente dallal $x$ : lequalzione precedente integralia da

$x=-\frac{1}{4 \Omega J\left(k+k_{-}\right)}\left[g^{*} \ln E(x)+g\left(k_{-}-k_{-}\right) E(x)-\frac{k-k}{2} E^{2}(x)+C\right] ;[19$

$C$ i la co-bante di integrazione. ele si determina potendo $x$-0:

$$
C=-g^{\prime \prime} \ln E(0)-g\left(k_{-}-k_{-}\right) E_{(0)+}+\frac{k_{-} k_{-}}{2} E^{2}(0)
$$

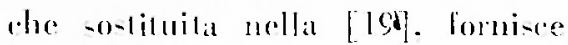

$$
\begin{gathered}
x=-\frac{1}{\left.1 . x J_{i}+k_{-}\right)}\left[g \cdot \ln \frac{E(x)}{E(0)}+g\left(k_{i}-k_{-}\right)(E(x)-E(0))-\right. \\
\left.-\frac{k_{-} k_{-}}{2}\left(E^{2}(x)-E^{*}(0)\right)\right] .
\end{gathered}
$$

validit per $x>0$.

Fiaminiamo landamento del campo seftrico nelle due regioni: nella prima eso de oviamente co-1ante, mantando le ratiche, nellit seconda è delerminalo, in funzione del valore $E(0)$ sullo strillo sede dell'azione ionizzante, dallat equaz. [21]. Risulta piin comodo porre la 121$]$ sollo forma leggermente differente, considerando come parametri il rapporto $u-E(x) E(0)$ e il rapporto $r-h E(0) g$ fra lo

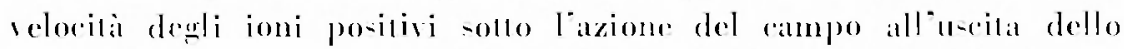
-lralo - lano e la velocila del vento. Si olliene rosi

$$
x=-\frac{g^{2}}{1 . \pi J(k+k)}\left[\ln u+\left(\frac{k}{k}-1\right) r(1-u)+\frac{k}{2 l_{i}} r^{2}\left(1-u^{2}\right)\right] \text {. }
$$

Volla -econda regione, cando posilivo il segno della densila di carica jerevalente, poiché grli ioni negativi ri-ulano diradali ri-pello at queili po-itivi a callsa della loro magniore velocilà il valore awoluto

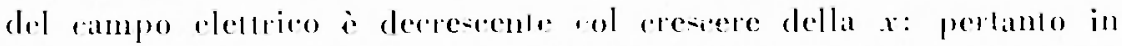
tale regione i verifial sempre $|E(x)|<\left|E_{t}(0)\right|$. quindi "rompleso Ira 0 and 1.

Per semplificare, potremo considerare due casi dipiei, il primo in rui la $g$ eia prosima al valore di ramsizione, dioe all'incirca $r$

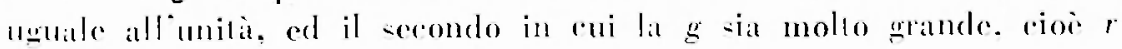


fendenlo a zaro. A-ommiamo per la $l$ il ralore $10^{-5}$ amp=3. $10^{-}$

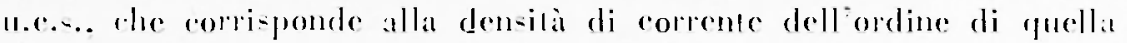

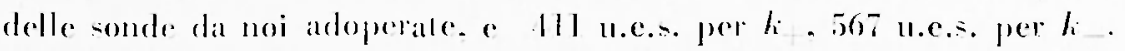

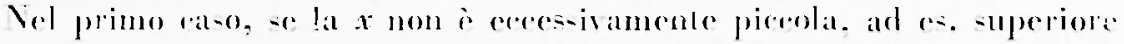

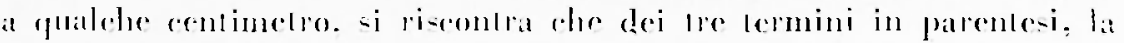

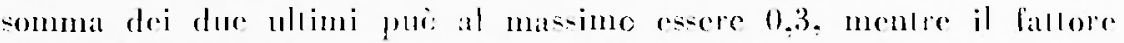

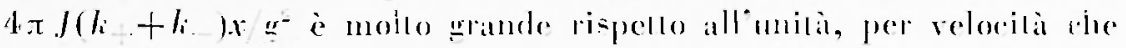
si agrgimo inlorno al valore sopua menzionalo. Perlanlo il lermine logarimieo ri-ula di gran lunga prevalente rispello agli altri due:

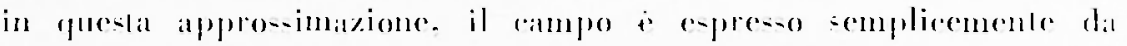

$$
E(x)=E(0) e^{-\frac{1 \pi J\left(k_{+}+k_{-}\right)}{g^{2}} x} \cdot
$$

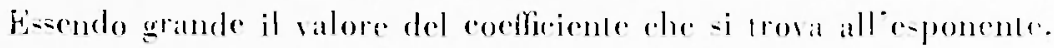

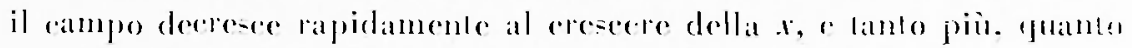
più prosima $i$ la velorili al valore di tran-ixione: il rampo puo silener-i nullo a brese distanza dallo stato sede dellazione ionizzanle. Per ogni valore della $x$, quando la velocila crese allonlanandosi dal

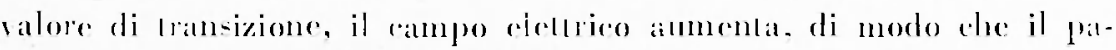

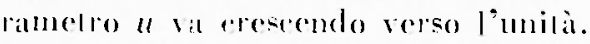

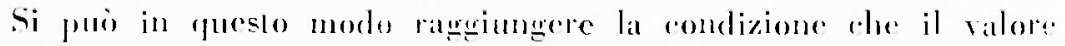
del lns non sia piì preponderanle rispello ai due remini -meessivi: dal pueso momenlo la prandezza dei tre termini diventa eomparabile. Per rendereene ragione oserviamo che nell`inorno di $u=1$ posiamo siluppere in serie il logarimo consorvando nello sviluppo solo il primo remine: abbiamo in pue-lo modo

$$
E(x)=E(0)\left\{1-\frac{\left.1-x J+h_{i}\right) x}{g^{2}\left[1-\left(\frac{l}{k}-1\right) r-\frac{1}{b} r^{2}\right.}\right\} \cdot[24]
$$

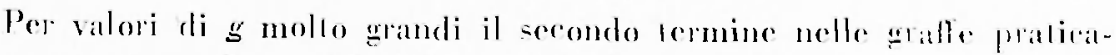

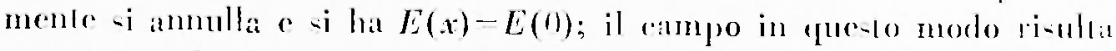
rostante anche lungo la seconda regione, poiche le ratriehe -ono rosi diradale da non far sentire piu la loro influenza.

11 gralico di fig. 5 indiea qualilativamente landamento del rampo elentroo in funzione della $x$ per un dialo valore della $g$ : fuesta curva

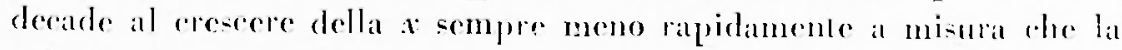
velocilit del vento diviene forte. 
Determiniano ora il potenziale asimto dallo - rialo inlermedio: al tale

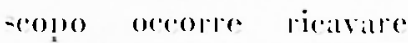
dal la [20l] la dillerenza di potenziale tra lo stratu $-1 e s=0$ e lia seconda almaturat.

lal $\lceil 29\rceil$ non it risolubile in remini funisi

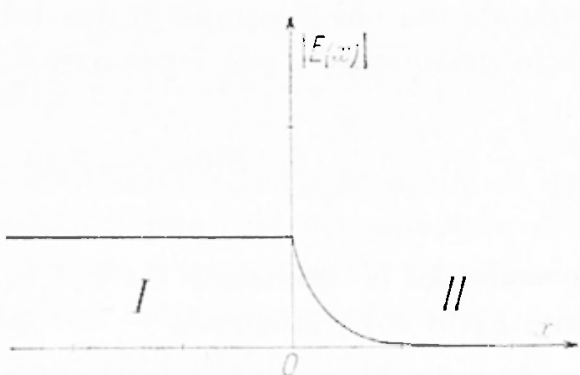

lit. 5 rispello allat $E$; pero con una intrerazione per parti, 2 posibile ricat sare il valole della differenza di polenziale $f_{2}$ in lunzione del campo; inlalli -i ha

$$
I_{2}=-\int_{0}^{2} l x d x=-[E x]_{0}^{T_{2}}+\int_{i}^{r_{2}} \mathrm{x} d E
$$

Eifelluanto loperazione. -i ottiene

$$
\begin{aligned}
& \left.V_{z}=\frac{1}{1 . \pi\left(l_{i}+l_{i}\right)}\right) g^{2}\left(E\left(l_{2}\right) E(0)\right)+g\left(\frac{l_{i}-l_{i}}{2}\right)\left(E_{2}\left(l_{z}\right)-E^{2}(0)\right)- \\
& -\frac{k-k^{\prime}}{3}\left(E^{3}\left(C_{2}\right)-E^{3}(0)\right) ?
\end{aligned}
$$

filimmanto il campe $E\left(l_{2}\right)$ fra la $\lfloor 26\rceil$ a lai $[21]$. dopo arer poilo in fuesta $x=l$, s olliene il valore della diflerenza di polenziale $\mathfrak{V}_{2}$ in funzione del rampo $E(0)$ sullo stalo intermedio o dei restanti pat-

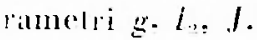

Per la forma della [2]], questa eliminazione non is eflelluabile in lemini fniti: per lramile del calcolo numerico, arendosi più parametri variabili da considerare, loperazione di caleolo riesere piut-

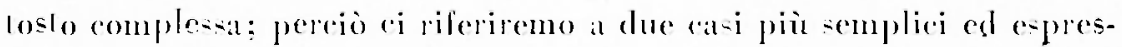
-ivi. e preatamente a quello in ani lat velocita id posima al valore

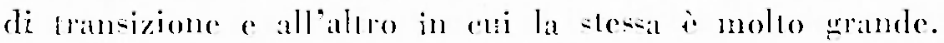

Vel primo eaco. se $a=E(l.) / f(0)$ i molto pireolo, powiano. nella

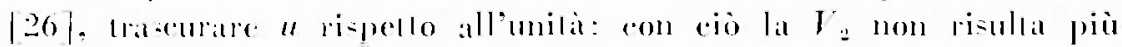

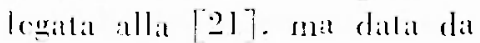

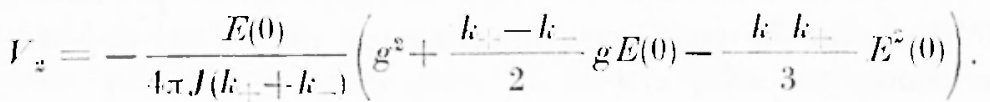


Rivordando ora che il campo ai due lati dello strato intermedio deve avere lo sesso valore, può esprimersi $E_{2}(0)$ in funzione di $l_{1}$ ció̀

$$
E(0)=-E_{0}=-\frac{V_{1}}{l_{1}} \quad,
$$

imponendo poi la condizione $V=V_{1}: V_{2}$ possono eliminarsi dalla precedente E.,iv) e $V_{2}$ oltenendo

$$
g^{2}-\frac{k_{-}-k_{-}}{2} \frac{V_{1}}{l_{i}} g-\frac{k_{-} k_{-}}{3}\left(\frac{V_{1}}{l_{1}}\right)^{2}-4 J J\left(k_{-}+k_{-}\right) l_{1}\left(\frac{V}{V_{1}}-1\right)=0 .
$$

Risolvendo rispetto alla gr, si ha

$g=\frac{V_{1}}{l_{1}} \frac{k_{-}-k_{+}}{4}\left[\sqrt{1+\frac{16}{3} \frac{k_{-}}{\left(k_{+}-k_{1}\right)^{2}}+64 \pi J \frac{k_{-}+k}{\left(k_{-}-k_{+}\right)^{2}}\left(\frac{l_{1}}{V_{1}}\right)^{3}\left(V-V_{1}\right)-1}\right]$

ove jer la radice si escelto il segno positivo dovendo risultare $g>0$. Determiniamo ora il valore di $g_{2}$, per il quale lo strato intermedio as-ume il potenziale della seconda armatura, cioè $V_{1}=V$ : si ha allora

$$
g_{2}=\frac{V}{l_{1}} \frac{k_{-}-k_{-}}{4}\left[\sqrt{1+\frac{16}{3} \frac{k_{-} k_{-}}{\left(k_{-}-k_{-}\right)^{2}}}-1\right]
$$

rorrispondentemente si ha $V_{n}=0$. Dal valore $V_{1}=V$, il potenziale diminuisce al erescere della velocità del vento dapprima lentamente e quindi tanto più rapidamente per kuanto il valore della $J$ è piccolo; con Lutto ciò tale dipendenza non complica l'andamento del fenomeno; infalti, per velocita g cosi forti, nell'interno dello strato intermedio potra ritenersi raggiunto il regime di salurazione e la $i$, a parita di intensilà di ionizzazione, risulterà praticamente costante ed indipendente dalla g.

Consideriamo ora il caso in cui la velocità del rento sia così grande e le cariche cosi diradale nella seconda regione, ehe il rapporto $u=E(l ..) / E(0)$ risulı prossimo all"unità. In questa eventualità vale per il campo eletrico la [24], whe fu da noi otlenuta rilenendo appunto trascurabile il termine $(1-u) / 2$ di fronte ad $1:$ per determinare il potenziale in questo caso integriamo la stessa $[24]$ rispetto and $x$, limitandola tra $x=l$, e $x=0$ od otlerremo 


$$
I_{z}=E_{0}^{\prime}: 1-\frac{2 \pi J\left(k_{-}+k_{-}\right)}{g^{2}\left[1-\left(\frac{k_{-}}{k_{-}}-1\right) r-\frac{k_{-}}{k_{+}} r^{2}\right]} l_{2}^{\prime} .
$$

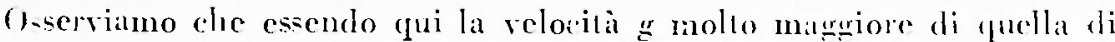
transizione, e quindi $r$ prossino a zero, nel denominalore della frazione posono trascurarsi il secondo e terzo termine rispetlo al primo. Allora la precedente si semplifica uleriormente c diventa

$$
V_{2}=E_{0} l_{2}\left(1-\frac{2 \pi J\left(k_{+}+k_{-}\right)}{g^{2}} l_{2}\right) \quad:
$$

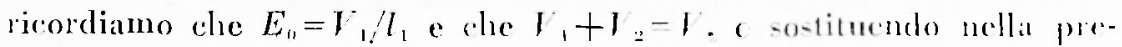
redente, si ha

$$
\boldsymbol{V}_{1}=\frac{l_{1} l_{1}}{l_{1}+l_{2}\left(1-\frac{2 \pi J\left(k_{+}+k_{-}\right)}{g^{2}} l_{2}\right)} .
$$

Se ne deduce che per $g$ tendente ad infinito it potenziale conde a quello statico $V l_{1}\left(l_{1}+l_{2}\right)$ in assenzal di cariche.

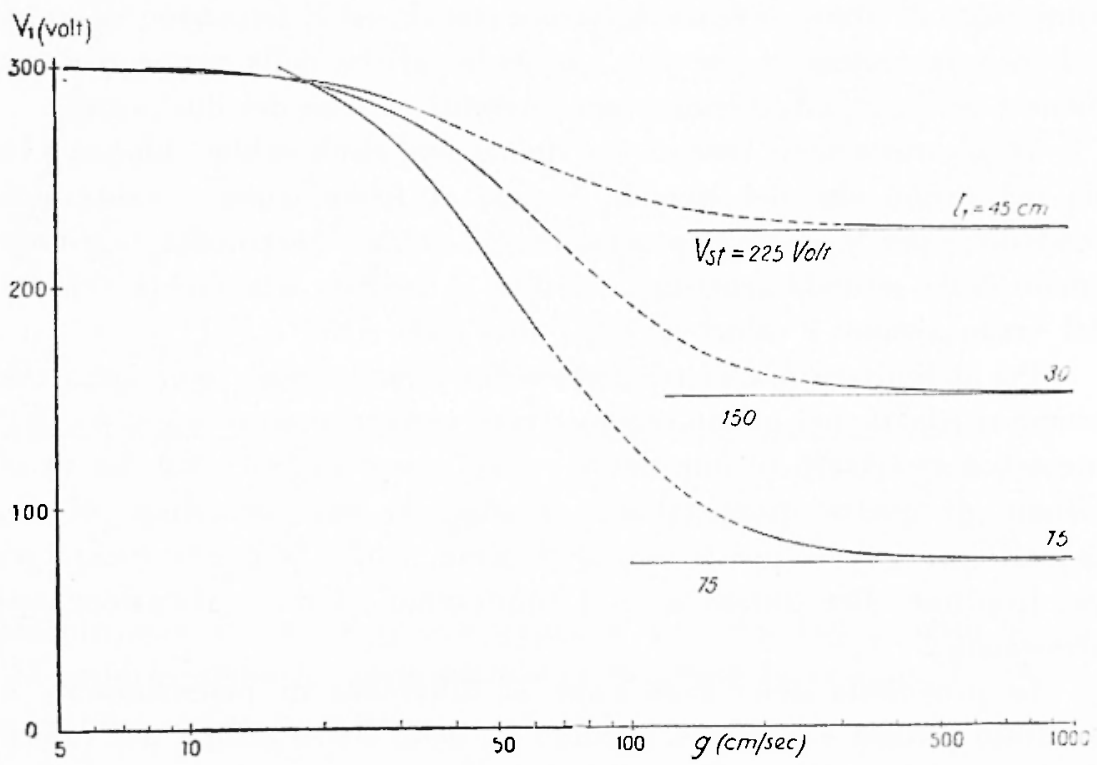

Fig. 6

Vél grafico di fig. 6 è riportato l'andamento di $r^{\prime}$ in fumzione di $g$ allorché lo stralo intermedio si trova a 15,30 e 45 rm di dislanza dalla prima armalura, per un valore della densita di corrente 
$I=3.10^{\circ}$ u.e.s. per ma differenza di potenziale $V=1$ u.e.s., deteminato per mezo delle fommle [30] [34] sui due lratti iniziali - Lerminali (tratto pieno), mentre i punti inlermedi sono tati delerminati per mezzo del calcolo numerieo eliminando, rome fu giat dello, il parametro $u$ tra le $[26]$ \& $[21]$.

Interesal ora di incllere a raflento i risultati ottenuli nelle trattaxioni relative al prino a al secondo renime di funzionanento.

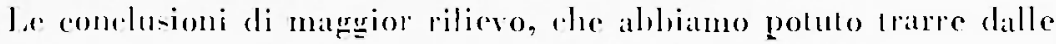

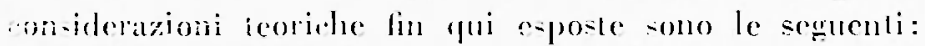

1) per velorita del rento rerenti a partire da zero, il polenziale dello trato sede dell"azione ionizzante - i spostal dal valore che sti compete nel condensatore in hatec alla preanza della carica spatxiale, rero valori canpre piì alti, e leade a ragrimgere il potenziale della seconda armallura. mantenemdosi il fenomeno sompre nel primo

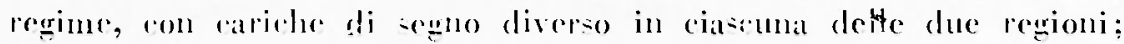

2) per velocili del vento -uperiori, il polenziale dello strato diminuiser di nuoro a tende al valore -lation, od il fenomeno si srolge nel secondo regime in eni ono assenti le cariche nella prima regione, menter nella seconda regione sono presenti andehe dei due segni.

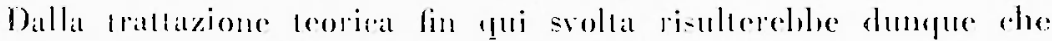
-ia nel primo che net seondo regrime di funzionamento esiste una eondizione per la quale il polenziale dello strato inlermedio raggiunge

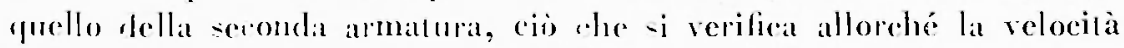
del vento atesume i ralori $g_{1}$ e g... dali dalle [15] e [31].

Per delimilare ora la transizione fra i due regrimi osecriano che

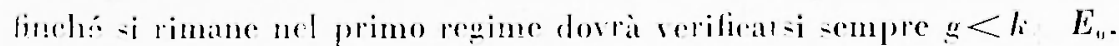

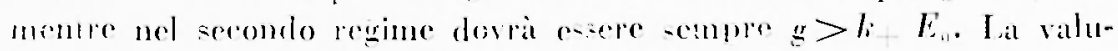
lazione di fue-te di-nguaglianze implieal la determinazione di $E_{n}$,

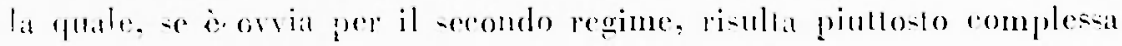

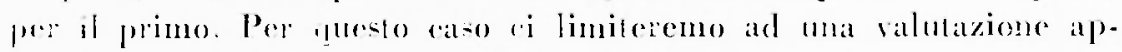
pron-antalit.

In prosimila della transizione ia dillerenza di potenziale $V_{2}$ e

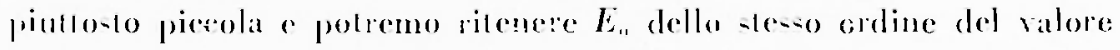
a reolute medio del campo nella seconda regione, pur mantenondo-i li. par la |z!. sempre minore:

$$
E_{0}<\frac{V_{2}}{l_{2}}=\frac{l-V_{1}}{l_{2}}
$$


Quindi funché si rimane nel primo regime, la velod:lit : non polua essere mai magriore di $h$. $(V--T, / l$.

Consideriamo il graffero di fig. 7 nel quale sono tractiate. per l'in.

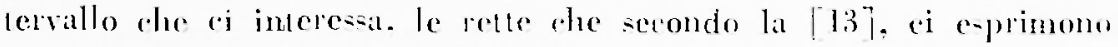
$1 /(1 / 0 / t)$

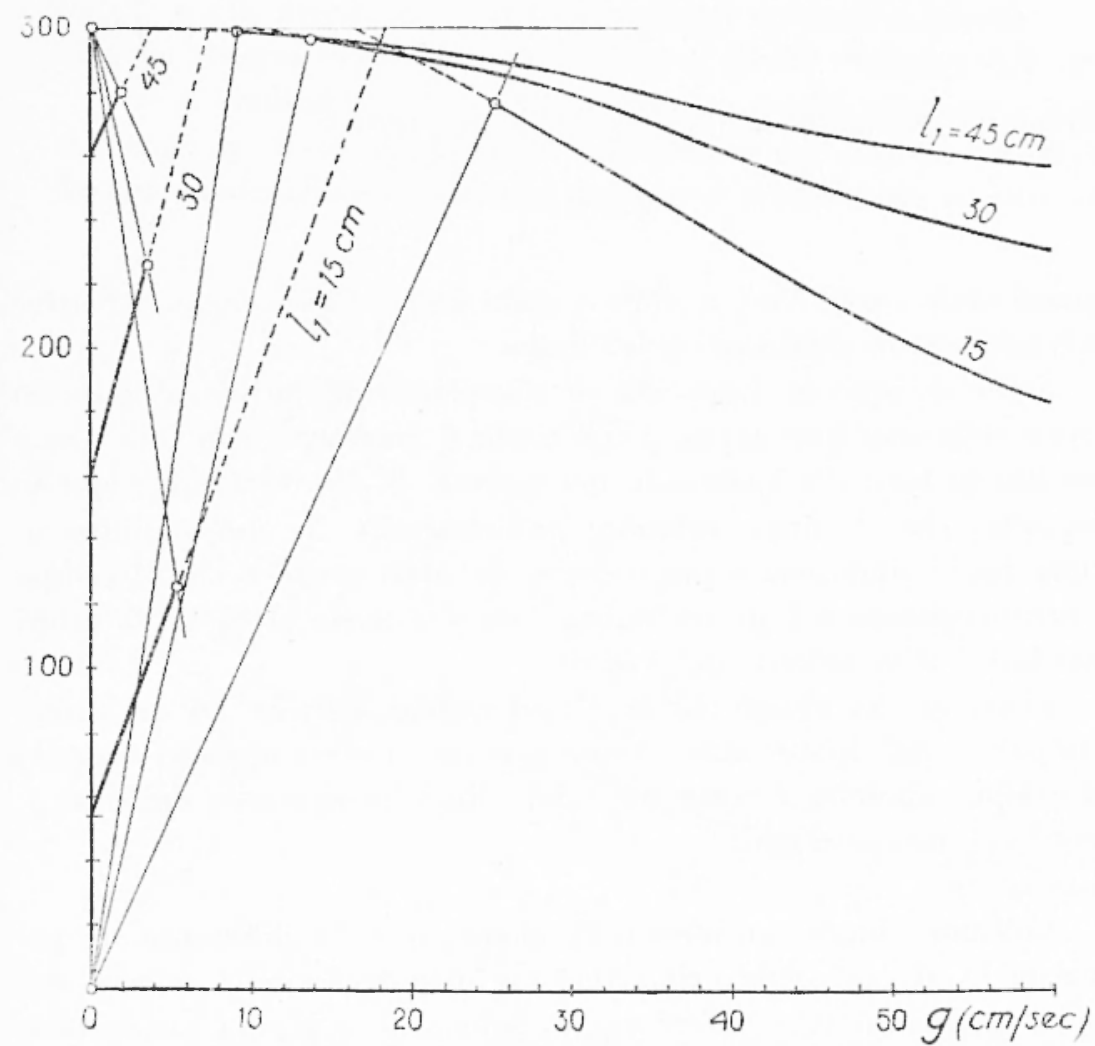

Fig. 7

l'anrlamento dellat $V_{i}(g)$ nel primo regime e le curre che. scomdo lat

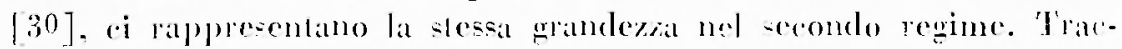
ciamo in tale grafleo le relle

$$
g=k \cdot \frac{V-I_{1}}{l_{2}}
$$

per i rispettivi valori $l_{1}=15,30$ e 15 cm. Tulli i punli delle relle $V_{1}(g)$ a destrat della intersezione non rilppresentano uno stato fisieo 
reale, in quanto la velocita del vento pisultereble piì rhe suffieiente al passagrio nal secondo regime.

Per il secondo regine invece, oserviamo ehe nella prima regione non esiste carica e quindi $E_{n}=V, l_{1}$. Pereio in questo regime dere ence semple

$$
g>i \frac{r_{1}}{i_{1}} .
$$

Tranciamo allalogamente nel grafico le the ictle

$$
g=t i \frac{l_{1}}{i_{1}} \text {. }
$$

I punti delle curve [30] a sinjstra delle intersezioni con le rispeltive rolle non hammo significato fisico reale.

E-isls dumpue un inlervallo di transizione dal primo al secondes regime relalivamente ampio, e nel quale il fenomeno non può essere descritlo in hase alle ipolesi da noi assunte. F' da osservare a guesto proposilo. che il limile inferiore dellinlervallo da noi slabilito in prima applossimazione è più elevalo che nella realla e che il rampo di funzionamento nel primo regrime non si esencle al di la di valori a-ail hassi della relocita del vento.

Rileviamo da ulimo che le con-iderazioni teoriche fin gui wolte smo partile dall'ipolesi che il renlo spirasse in verso opposto a fuello del campo: allorehe il verso del vento roincide ron fuello del rampo. i fianlati -0 ono analoghi.

thbiamo sinora ron-islerato l'andamento della differenza di poImziale $V_{1}$ a i rapi della prima regione in fumzione della velocià del vento, in quanto lale differenza di potenziale e quella che meglio rappresenta, dal punlo di visa fisico, landamento del fenomeno.

Per una valulazione dellinfluenza del vento sul polenziale as-unto dallo stalo sede dellazione ionizzante in confromto al valore rhe gli -pellerehbe in rondizioni satiche, in a-conza cioe di cariche pertur-

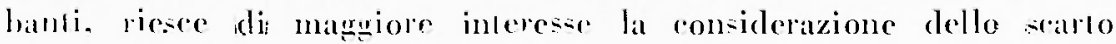
relalivo fra il polenziale $V_{1}$ ed il polenziale slatico $T_{+t}-T l_{1} l$ rioi $\Delta V, V=\left(I, \ldots, V_{-1}\right) I_{s t}$.

Vella fig. 8 alhhiamo riportato l'andanento di tale satrto in funzione della velocili g per le tre solile posizioni dello strato inlermedio fra le almalue del condentalore, e cioe $l_{1}=15,30,45 \mathrm{~cm}$, nel a-o) dal noi -ludialo di $l=60$ (.m. $Y=1$ u.e.s. e $J=3.10^{-2}$ u.e.s.; it 
grafico es salo tracediato in hase allar formula $[13]$ per il primo rewime ed alle $[30]$ e $[34]$ per il serondo regime. Nel prafieo non rompare la zona relativa all'intervallo di transizione. eui non si celende lat validisi fisica delle formule; inoltre la scala adoltata in ordinsta ${ }^{\circ}$ più ristretla di quella del corrispondente diatramma sperimentale di fig. 2, e riò perché gli scarti delerminali sperimentalmente si manlengono sompre notcvolmente inforiori a quelli risultanti dal ralcolo.

Se si confrontil questo diagramma con quello ollenulo sperimenlalmente, si rileva ma notevole somiglianza nei rispettivi andamenti. La schematizzazione lcorica del fenomeno, l'aver fatto atrixione dal-

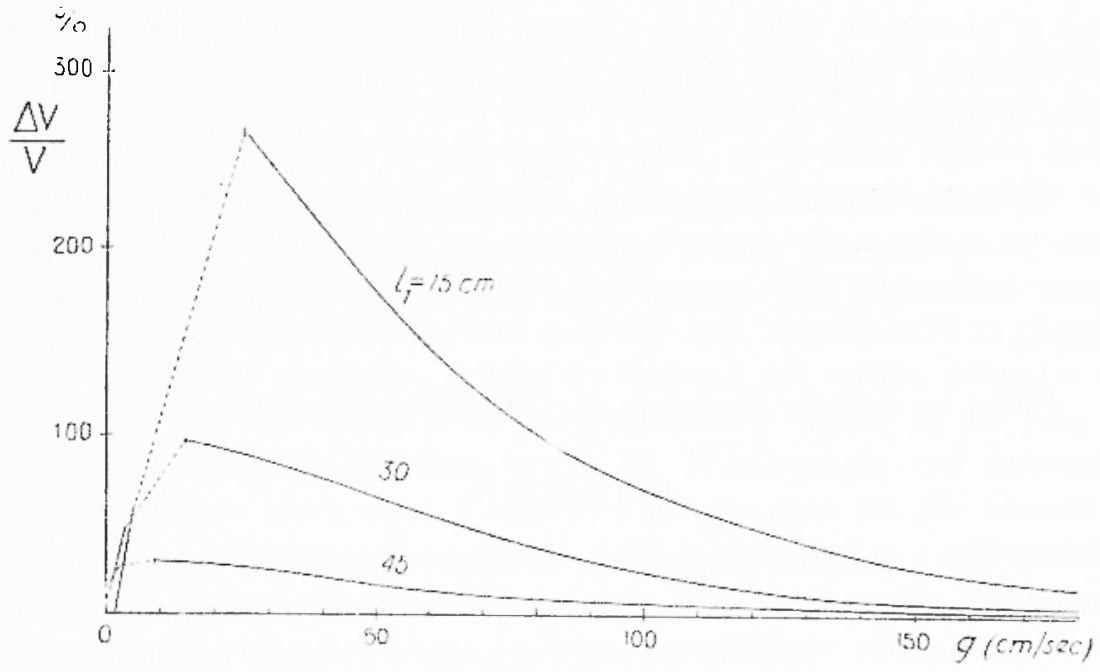

Fig. 8

l'effotliva exsenza dellat diffusione e le approssimazioni di rolta in volta adoltale per consontire ma tratlarione relalivanente sempliee: non alterano dunque le ciralleristiche esenziali del fenomeno stesso, il quale, almeno qualialivamente, risulta soddisfacenlemente interpretalo. Infati lesistenza di un intervallo di velocilà del vento in rui lo searlo ra cresendo al aresere della velocilà, il maniferlar-i di un massimo rhe, per le poszioni delto stralo intermedio da noi scolle, rade nell'intervallo compreso fra eirat $15 \mathrm{e} 30 \mathrm{~cm} / \mathrm{sec}$ e il suscownente diminuire dello seato steso cou lubleriore aumenlo della velocili del vento, trovano la loro eonferma negli andamenti determinabili teoricamente, in corrispondenza al primo ed al serondo regime.

La posizione in rui cade, cerondo la teoria, il valore massimo dello 
sarto -i trosa presmmibilmente ai confeni o nellontervallo di llansi-

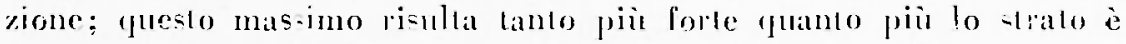
prossimo alla prima ammatura: secondo i dati rilevati dalla esparienza i valori appaiono alguanto minori di fuelli suggeriti dalla leoria: feraltro non bisogna dinentiare cone l'espericnza sia slata nesessariamente condota ron una sonda reale di estensione molto pirecola, mentre la teoria si hasa sulla considerazione di uno strato sede dell'azione ionizzante di e-tensione inciefnita. Lal divergenza lia andamento teorico e sperimentale puì cescre altribuita al fatlo rle, nelle

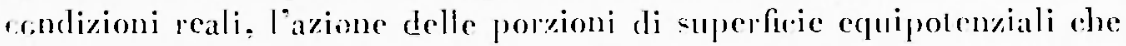
rimangono imperlurbate influise stll"andausento delle sle-se superficic in prossimita della sonda: guesto lallo del resto fu o-servato gria

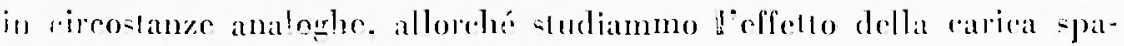
ziale jn maneanza di rento.

E' lecito rilenere she i falti acecrati con fueste ricerche e la loro inlerpretazione teorica polrammo dare una guida per una più

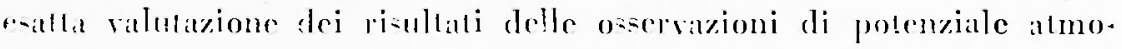
-forico.

Roma - Istituto Vazionale di Geoficia -... Sollembre 1918.

\section{$R I$ IST( iTi)}

Gli t 1. espongono alcume loro ricerche condolle per studiare l'influenza del renlo sulla misura del polenziale oletarien per mezao delle sonde radioatlive. Vongono daprima descrilli i risultati stlomuli nella misura del potenziale slesso in an rampo miforme nel quale fluisere,

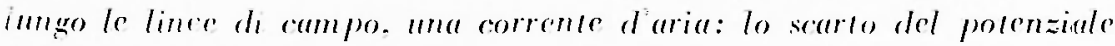
dal suo valore slatico cresce dapprma fino at un massimo al crescere. della velocitì de! vento, e diminuisece sllecessivamente, lendendo asinbotiramerale a zero. Tiene poi stiluppala man trallazione teorica per mezan atr!la quale possono essere sorblisfacentemente inlerprelati i risullati sperimenlali: dal!a stessa risulla che debbono essere distinli nel fenomeno due dieresi regimi di funsionamento a seconda che la

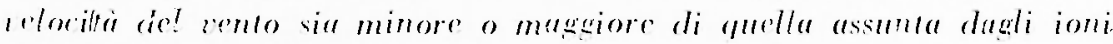
sollo l'asione del rampo: tali due regimi corrispondono ai tralli in

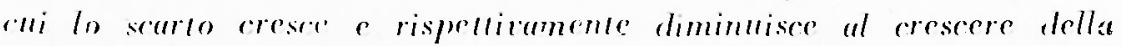
irlocicie ad wrilo. 